\title{
A SURVEY OF EARLY-TYPE STARS BASED ON THE TWO MICRON ALL SKY SURVEY DATABASE ${ }^{1}$
}

\author{
R. ORTIZ \\ Escola de Artes, Ciências e Humanidades, Universidade de São Paulo, Avenida Arlindo Bettio 1000, \\ 03828-000 São Paulo, SP, Brazil; ortiz@astro.iag.usp.br \\ M. Malacarne \\ Departamento de Física, Universidade Federal do Espírito Santo, Avenida Fernando Ferrari 514, 29060-900 Vitória, ES, Brazil \\ R. WILHELM \\ Department of Physics, Texas Tech University, Campus Box 41051, Lubbock, TX 79409, USA \\ R. D. D. Costa, S. Rossi, and W. J. Maciel \\ Departamento de Astronomia, Instituto de Astronomia, Geofísica e Ciências Atmosféricas, Universidade de São Paulo, \\ Rua do Matão 1226, Cidade Universitária, 05508-900 São Paulo, SP, Brazil \\ AND \\ A. F. M. Costa \\ Instituto de Física, Universidade Federal do Rio Grande do Sul, 91501-900 Porto Alegre, RS, Brazil \\ Received 2007 March 13; accepted 2007 June 11
}

\begin{abstract}
We report the results of a spectroscopic survey of blue stars near the south Galactic pole, selected according to their color indices in the 2MASS database. The main scope of this work is to determine the nature of the objects that comprise the sample. Some characteristics of the $\mathrm{H} \beta, \mathrm{H} \gamma$, and $\mathrm{H} \delta$ lines which are sensitive to temperature and gravity were measured and eventually compared with a grid of model atmospheres to obtain $T_{\text {eff }}$ and $\log g$. In some cases the data allowed a unique, reliable classification of the star. Among the 44 stars studied, 5 objects are reliably classified as subdwarfs, 12 as A-type stars, and 3 as field horizontal-branch (FHB) stars. The remaining objects are either A or FHB stars, but additional observations are necessary to distinguish between these two types. Neither degenerate nor lowgravity stars (post-AGB) have been identified in the sample.
\end{abstract}

Key words: Galaxy: halo — Galaxy: stellar content — stars: early-type — stars: horizontal-branch — subdwarfs — surveys

\section{INTRODUCTION}

The Galactic poles have long been explored in the quest for halo objects, especially field horizontal-branch (FHB) stars, including RR Lyrae variable stars. Because the absolute magnitudes of HB stars are in a narrow range (Gratton 1998), they can be used as standard candles to figure out the spatial distribution of stars in the halo (Sluis \& Arnold 1998). Numerous surveys have been carried out to identify halo members, which can be gathered into two main groups: the photometric surveys, which make use of Strömgren and/or broadband photometry (Green et al. 1986; Noguchi et al. 1980; Stobie et al. 1987; Kilkenny et al. 1991), and the objective-prism surveys, which classify stars based on very low resolution spectra (Sanduleak \& Pesch 1987; Sanduleak 1988). More recently, the completion of major CCD and infrared surveys has opened a new perspective in identifying blue stars in deep fields. The Sloan Digital Sky Survey, for example (York et al. 2000), covered a quarter of the sky in five colors. It has been used to identify white dwarfs (Harris et al. 2003) and blue horizontal-branch (BHB) stars at distances from 7 to $60 \mathrm{kpc}$ from the Sun (Sirko et al. 2004). This lower limit of distance of BHB stars is caused by the saturation magnitude of the Sloan survey at $g=15.5$. The infrared surveys DENIS and the Two Micron All Sky Survey (2MASS) constitute another important database to identify hot, bright stars far from the Galactic plane.

\footnotetext{
${ }^{1}$ Based on observations obtained at the Pico dos Dias Observatory, operated by the National Laboratory for Astrophysics, Brazil.
}

Ortiz (2002) and Brown et al. (2003, 2004), among others, have identified BHB stars, young stars, and other kinds of "blue" objects in these infrared surveys.

The discovery of main-sequence (MS) stars of early spectral type far from the Galactic plane is perhaps more remarkable than the identification of BHB stars. A few hundred MS stars of spectral types A and B have been identified so far (Conlon et al. 1990; Mitchell et al. 1998; Pier 1982, 1983), but it is not clear whether they have been ejected from the Galactic plane by collisions (Conlon et al. 1990) or whether they were formed in the halo itself (Conlon et al. 1992). Spectroscopic surveys can function as an effective tool to find more objects of this kind.

Unfortunately, the usage of color indices to classify stars is not very reliable. For instance, A and B MS stars show broadband color indices very similar to those of BHB stars. Brown et al. (2004) claim that BHB stars constitute only half the stars selected according to their infrared color indices. As an alternative, Strömgren photometry can be used more reliably (Stetson 1991; Flynn et al. 1994). Another good method is to measure the equivalent width of the Balmer lines, especially $\mathrm{H} \delta$. The $D_{0.2}$ method (Pier 1983; Sommer-Larsen \& Christensen 1986; Wilhelm et al. 1999a) also allows fine discrimination among the several kinds of blue stars, although the separation between BHB and MS stars remains unclear in many cases (Beers et al. 1992; Sirko et al. 2004). At stellar temperatures $T_{\text {eff }}>10,000 \mathrm{~K}$ it becomes extremely difficult to separate BHB and MS stars, because the surface gravity of BHB stars increases to MS gravities in this regime. The highresolution work by Behr (2003) on BHB stars in various globular 
clusters illustrates this effect. In Figure 9 of that paper the extended HB of various clusters quickly approaches $\log g$ values typical of MS stars $(\log g \simeq 4.0)$. These data are also consistent with the theoretical zero-age HB by Dorman et al. (1993), which predicts a similar increase in $\log g$. Therefore, it becomes nearly impossible for stars hotter than $10,000 \mathrm{~K}$ to separate the BHB from the MS using surface gravity as an indicator.

In this work we present the results of an infrared, flux-limited spectroscopic survey of the south Galactic cap, based on the 2MASS point-source catalog. The survey is especially aimed at stars that have been overlooked in previous surveys of this kind. Stars are classified according to their visual spectra, and some of their atmospheric parameters are determined. In $\S 2$ the criteria used to select the sample are described; $\S 3$ describes the observations; $\oint 4$ is devoted to presenting our results, including a summary of the techniques used to determine the nature of the stars; and our conclusions are presented in $\S 5$.

\section{THE SAMPLE}

\subsection{JHK Colors As a Tool to Select Early-Type Stars}

The 2MASS (Skrutskie et al. 1997) covered the whole sky at three wavelengths, $J(\lambda=1.25 \mu \mathrm{m}), H(\lambda=1.65 \mu \mathrm{m})$, and $K(\lambda=2.15 \mu \mathrm{m})$, and is claimed to be complete up to magnitudes of $15.8,15.1$, and 14.3, respectively. The point-source catalog constitutes a fairly uniform photometric database, since each hemisphere was fully observed with the same set of telescopes, filters, and detectors. In this work we use the 2MASS catalog to select sources showing color temperatures greater than $10,000 \mathrm{~K}$. Figure 1 shows a sample consisting of about 12,500 stars near the south Galactic pole together with intrinsic color indices of dwarfs calculated by Bessell \& Brett (1988). The density of points does not represent the statistical distribution of colors, but it emphasizes the points near the lower left corner of the figure. These diagrams show that the MS runs almost perpendicular to the $H-K$ axis. Therefore, this color index is not very reliable for distinguishing stars by their temperature. On the other hand, both the $J-K$ and $J-H$ color indices show more explicit dependence on $T$. We are aware that, whatever the infrared color indices used, they do not constitute an accurate method to determine the temperature, because the spectral energy distribution approaches the RayleighJeans regime in the near-infrared. Nevertheless, this tool allows a rough selection of hot stars in large databases such as 2MASS, and the temperature can eventually be estimated based on spectral features of the observed stars.

\subsection{Criteria for Selecting Early-Type Stars Near the South Galactic Pole with Good-Quality 2MASS Photometry}

Once the $J-K$ and $J-H$ color indices have been chosen to select infrared sources according to their color temperature, additional selection criteria had to be adopted in order to select only sources that have good photometric measurements. A summary of the criteria used in this work is as follows:

1. $b<-60^{\circ}$,

2. $J-H \leq 0.0$ and $J-K \leq 0.0$,

3. $J \leq 15.8, H \leq 15.1$, and $K_{\text {short }} \leq 14.3$,

4. ph_qual flag $\neq X, U, F, E$ in the three bands.

The limitation in magnitude aims to select only sources within the completeness limit of the survey, as well as to avoid sources which would require too long an integration time when observed with a small telescope. The flags that accompany the magnitudes in the 2MASS catalog give information about problems that might have occurred during the measurements or the data reduction. The
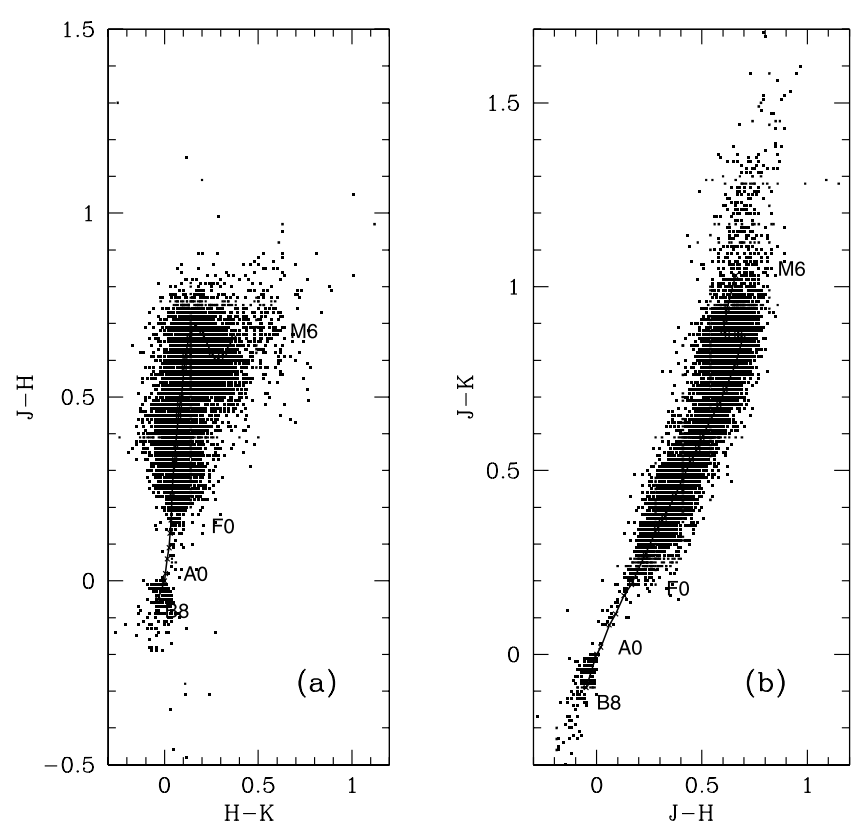

FIG. 1.-The 2MASS sources near the Galactic pole. The symbols represent sources with good photometry, according to the flags of the 2MASS point-source catalog. The solid line represents the standardized colors of MS stars according to Bessell \& Brett (1988), identified by their spectral types along the curve. The density of points does not represent the statistical distribution in color, but it was made to emphasize the population of objects with $J-H<0, H-K<0$, and $J-K<0$.

rejection of sources with bad ph_qual flags aimed to exclude stars which had problems with their photometry during the data reduction. The meaning of the flags noted above is as follows: $\mathrm{X}$ indicates that there is a detection, but no useful brightness estimate can be extracted using any algorithm; $U$ indicates an upper limit on the magnitude; F designates either aperture photometry or a saturated source; and $\mathrm{E}$ indicates that either the quality of the profile-fit photometry is very poor or the profile-fit photometry does not converge, and an aperture magnitude is reported. On the other hand, sources with flags B and $\mathrm{C}$ have been included because their fluxes are reliable, although with smaller signal-to-noise ratios ( $\mathrm{S} / \mathrm{N}=7$ and 5, respectively). According to the 2MASS Survey Strategy Requirements, version 1.7 (Cutri et al. 2003), the restrictions of the limiting magnitudes of the three bands above correspond to $\mathrm{S} / \mathrm{N} \geq 10$. However, a few cases of sources showing photometric flags B, C, and D have been noted; thus, the values concerning the $\mathrm{S} / \mathrm{Ns}$ must be considered approximate.

Despite all the precautions taken in the 2MASS reduction pipeline, a few problems persist with the photometry. About $20 \%$ of the sources selected according to the criteria specified above are in fact optical pairs or binaries. Although the bl_flag of these sources indicates that the components are not blended and the photometric method used was profile fitting, their $J H K$ color indices are incompatible with the spectral type of the stars. A search in the SIMBAD database shows that their spectral types are generally F or later. In order to avoid such objects, all sources which appear double in the Digitized Sky Survey were removed from the observation list.

The $J H K$ error bars are the main source of error in the determination of spectral type. On average, they are $0.03,0.05$, and $0.07 \mathrm{mag}$ in the $J, H$, and $K$ bands, respectively, for magnitudes greater than 14.0, which is near the limit considered for the $K$ band. Consequently, the errors in the color indices are $\sigma(J-H)=0.06$ and $\sigma(J-K)=0.08 \mathrm{mag}$, which represent five spectral subclasses for both indices. This means that even stars of A5 spectral type may show $(J-H)$ and $(J-K)$ near zero and may be 
TABLE 1

2MASS Data of the Sample Observed during This Survey

\begin{tabular}{|c|c|c|c|c|c|c|c|c|c|c|c|c|}
\hline $\begin{array}{l}\text { Number } \\
\text { (1) }\end{array}$ & $\begin{array}{l}\text { 2MASS Name } \\
\text { (2) }\end{array}$ & $\begin{array}{c}l \\
(3)\end{array}$ & $\begin{array}{c}b \\
(4)\end{array}$ & $\begin{array}{c}\text { Blue Mag. } \\
\text { (5) }\end{array}$ & $\begin{array}{l}\text { Red Mag. } \\
\text { (6) }\end{array}$ & $\begin{array}{c}J \\
(7)\end{array}$ & $\begin{array}{l}\sigma_{J} \\
(8)\end{array}$ & $\begin{array}{l}H \\
(9)\end{array}$ & $\begin{array}{c}\sigma_{H} \\
(10)\end{array}$ & $\begin{array}{c}K \\
(11)\end{array}$ & $\begin{array}{c}\sigma_{K} \\
(12)\end{array}$ & $\begin{array}{c}B-V \\
(13)\end{array}$ \\
\hline $1 \ldots \ldots$. & $22361663-3142130$ & 15.861 & -60.332 & 12.70 & 13.80 & 13.834 & 0.029 & 14.021 & 0.047 & 14.059 & 0.055 & -0.2 \\
\hline $2 \ldots \ldots \ldots \ldots \ldots \ldots$ & $22385421-2352209$ & 31.670 & -60.003 & 11.57 & 11.94 & 12.180 & 0.024 & 12.254 & 0.025 & 12.297 & 0.023 & -0.07 \\
\hline $3 \ldots \ldots \ldots \ldots \ldots \ldots \ldots$ & $22393762-2455025$ & 29.689 & -60.389 & 12.50 & 12.80 & 12.708 & 0.023 & 12.721 & 0.022 & 12.788 & 0.025 & -0.21 \\
\hline $4, \ldots \ldots \ldots \ldots \ldots \ldots$ & $22475352-3829408$ & 1.278 & -61.836 & 14.10 & 16.30 & 13.902 & 0.032 & 13.921 & 0.044 & 13.969 & 0.063 & $\ldots$ \\
\hline $5 \ldots \ldots \ldots \ldots \ldots \ldots$ & $22480188-3503104$ & 8.503 & -62.543 & 12.33 & 12.29 & 12.770 & 0.024 & 12.825 & 0.025 & 12.842 & 0.030 & $\ldots$ \\
\hline 6.................... & $22501122-3759588$ & 2.064 & -62.396 & 13.90 & 14.20 & 14.050 & 0.029 & 14.071 & 0.040 & 14.087 & 0.069 & $\ldots$ \\
\hline 7 7................... & $22515349-4355577$ & 350.090 & -60.699 & 12.80 & 14.30 & 13.569 & 0.027 & 13.595 & 0.022 & 13.645 & 0.049 & $\ldots$ \\
\hline $8 \ldots \ldots \ldots \ldots \ldots \ldots$ & $22524524-2147476$ & 37.585 & -62.539 & 12.10 & 12.37 & 12.427 & 0.027 & 12.473 & 0.026 & 12.459 & 0.026 & $\ldots$ \\
\hline $9 \ldots \ldots \ldots \ldots \ldots \ldots$ & $22533111-2005016$ & 41.204 & -62.157 & 11.09 & 10.87 & 10.746 & 0.022 & 10.748 & 0.024 & 10.752 & 0.021 & +0.2 \\
\hline $10 \ldots \ldots \ldots \ldots \ldots \ldots$ & $22574779-1822567$ & 45.413 & -62.456 & 11.83 & 11.91 & 12.209 & 0.026 & 12.323 & 0.028 & 12.317 & 0.030 & $\ldots$ \\
\hline $11 \ldots \ldots \ldots \ldots \ldots \ldots$ & $22575321-3250528$ & 12.887 & -64.838 & 12.21 & 12.53 & 12.340 & 0.029 & 12.344 & 0.024 & 12.358 & 0.029 & $\ldots$ \\
\hline $12 \ldots \ldots \ldots \ldots \ldots \ldots$ & $23010311-1805337$ & 46.680 & -63.043 & 11.41 & 11.38 & 11.377 & 0.022 & 11.391 & 0.025 & 11.424 & 0.026 & $\ldots$ \\
\hline $13 \ldots \ldots \ldots \ldots \ldots \ldots$ & $23071216-3157380$ & 14.589 & -66.880 & 12.00 & 12.00 & 12.434 & 0.024 & 12.477 & 0.023 & 12.448 & 0.023 & $\ldots$ \\
\hline $14 \ldots \ldots \ldots \ldots \ldots \ldots$ & $23091568-4123105$ & 352.084 & -64.679 & 11.39 & 11.28 & 11.169 & 0.021 & 11.211 & 0.024 & 11.186 & 0.021 & +0.1 \\
\hline $15 \ldots \ldots \ldots \ldots \ldots \ldots$ & $23223143-4559463$ & 340.077 & -64.200 & 12.05 & 12.29 & 12.531 & 0.022 & 12.558 & 0.026 & 12.582 & 0.023 & -0.04 \\
\hline $16 \ldots \ldots \ldots \ldots \ldots$ & $23270592-4009350$ & 350.787 & -68.234 & 13.90 & 13.30 & 13.177 & 0.023 & 13.203 & 0.026 & 13.244 & 0.033 & $\ldots$ \\
\hline $17 \ldots \ldots \ldots \ldots \ldots$ & $23285991-1437024$ & 61.878 & -67.093 & 12.40 & 12.60 & 12.653 & 0.024 & 12.665 & 0.028 & 12.691 & 0.034 & $\ldots$ \\
\hline $18 \ldots \ldots \ldots \ldots \ldots \ldots$ & $23334736-3853280$ & 352.038 & -69.988 & 11.57 & 11.51 & 11.489 & 0.023 & 11.531 & 0.023 & 11.544 & 0.024 & $\ldots$ \\
\hline $19 \ldots \ldots \ldots \ldots \ldots \ldots$ & $23462825-3455590$ & 359.615 & -74.074 & 13.50 & 14.90 & 14.123 & 0.026 & 14.139 & 0.040 & 14.175 & 0.077 & +0.03 \\
\hline $20 \ldots \ldots$. & $23521950-3603516$ & 353.839 & -74.593 & 14.20 & 14.50 & 13.891 & 0.030 & 13.923 & 0.041 & 13.926 & 0.062 & +0.03 \\
\hline 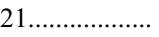 & $23591014-4342306$ & 332.011 & -70.350 & 12.18 & 12.29 & 12.209 & 0.024 & 12.226 & 0.025 & 12.213 & 0.024 & $\ldots$ \\
\hline $22 \ldots \ldots \ldots \ldots \ldots \ldots$ & $00152941-2105497$ & 67.347 & -79.825 & 12.15 & 12.40 & 12.242 & 0.023 & 12.316 & 0.025 & 12.326 & 0.023 & $\ldots$ \\
\hline $23 \ldots \ldots \ldots \ldots \ldots \ldots$ & $00284278-1823502$ & 90.819 & -79.822 & 12.13 & 12.31 & 12.313 & 0.027 & 12.330 & 0.024 & 12.367 & 0.029 & $\ldots$ \\
\hline $24 \ldots \ldots \ldots \ldots \ldots \ldots$ & $00284842-3632024$ & 328.549 & -79.443 & 13.50 & 13.90 & 13.686 & 0.032 & 13.717 & 0.029 & 13.772 & 0.060 & $\ldots$ \\
\hline $25 \ldots \ldots \ldots$ & $00335405-4547026$ & 312.367 & -71.022 & 13.70 & 14.50 & 14.143 & 0.034 & 14.146 & 0.051 & 14.252 & 0.073 & +0.1 \\
\hline 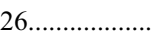 & $00383181-3913183$ & 314.660 & -77.611 & 12.70 & 13.00 & 12.839 & 0.026 & 12.844 & 0.030 & 12.855 & 0.037 & $\ldots$ \\
\hline $27 \ldots \ldots$ & $00471767-1453172$ & 118.219 & -77.722 & 13.20 & 14.00 & 14.110 & 0.032 & 14.132 & 0.033 & 14.199 & 0.064 & $\ldots$ \\
\hline $28 \ldots \ldots \ldots \ldots \ldots \ldots$ & $00480632-4439565$ & 304.897 & -72.450 & 12.49 & 12.80 & 12.658 & 0.024 & 12.715 & 0.028 & 12.701 & 0.030 & $\ldots$ \\
\hline $29 \ldots \ldots \ldots \ldots \ldots$ & $01011757-3342454$ & 285.662 & -83.082 & $\ldots$ & $\ldots$ & 13.232 & 0.026 & 13.361 & 0.028 & 13.431 & 0.043 & -0.18 \\
\hline $30 \ldots \ldots \ldots \ldots \ldots$ & $01090411-3307403$ & 271.583 & -82.894 & 11.91 & 12.03 & 12.444 & 0.022 & 12.570 & 0.028 & 12.621 & 0.037 & $\ldots$ \\
\hline 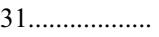 & $01111697-2138541$ & 163.494 & -82.899 & 12.10 & 13.30 & 12.920 & 0.023 & 12.961 & 0.025 & 12.996 & 0.029 & $\ldots$ \\
\hline $32 \ldots \ldots \ldots \ldots \ldots \ldots$ & $01142378-4710404$ & 291.766 & -69.450 & 12.48 & 12.59 & 12.365 & 0.024 & 12.382 & 0.023 & 12.379 & 0.026 & $\ldots$ \\
\hline $33 \ldots \ldots \ldots \ldots \ldots \ldots$ & $01294009-4015539$ & 274.303 & -74.667 & 11.71 & 11.73 & 11.441 & 0.026 & 11.459 & 0.023 & 11.443 & 0.023 & $\ldots$ \\
\hline $34 \ldots \ldots \ldots \ldots \ldots \ldots$ & $01511189-1732518$ & 181.245 & -73.210 & 11.68 & 11.46 & 11.578 & 0.023 & 11.610 & 0.023 & 11.617 & 0.026 & $\ldots$ \\
\hline $35 \ldots \ldots \ldots \ldots \ldots$ & $02165824-3447583$ & 240.943 & -70.177 & 12.70 & 13.20 & 13.049 & 0.026 & 13.106 & 0.029 & 13.091 & 0.034 & $\ldots$ \\
\hline $36 \ldots \ldots \ldots \ldots \ldots \ldots$ & $02192738-3637583$ & 245.464 & -69.107 & 12.40 & 13.00 & 12.665 & 0.023 & 12.696 & 0.029 & 12.701 & 0.029 & $\ldots$ \\
\hline 37................. & $02205977-3423353$ & 239.155 & -69.490 & 13.00 & 13.50 & 13.990 & 0.030 & 14.097 & 0.032 & 14.209 & 0.072 & $\ldots$ \\
\hline $38 \ldots \ldots \ldots \ldots \ldots \ldots$ & $02221984-2324558$ & 207.729 & -69.149 & 11.78 & 11.99 & 12.616 & 0.026 & 12.748 & 0.021 & 12.842 & 0.031 & $\ldots$ \\
\hline $39 \ldots \ldots \ldots \ldots \ldots \ldots$ & $02251836-2433280$ & 211.264 & -68.755 & 12.60 & 15.20 & 12.708 & 0.023 & 12.721 & 0.022 & 12.788 & 0.025 & $\ldots$ \\
\hline $40 \ldots \ldots \ldots \ldots \ldots \ldots$ & $02272918-3953377$ & 251.756 & -66.375 & 11.83 & 12.07 & 12.197 & 0.024 & 12.212 & 0.026 & 12.288 & 0.024 & $\ldots$ \\
\hline $41 \ldots \ldots \ldots \ldots \ldots$ & $02324780-2038431$ & 202.761 & -65.990 & 13.30 & 13.40 & 13.455 & 0.026 & 13.509 & 0.034 & 13.521 & 0.043 & $\ldots$ \\
\hline $42 \ldots \ldots \ldots \ldots \ldots \ldots$ & $02344423-2108454$ & 204.290 & -65.735 & 12.00 & 12.10 & 12.291 & 0.026 & 12.299 & 0.025 & 12.297 & 0.026 & +0.01 \\
\hline $43 \ldots \ldots \ldots \ldots \ldots$ & $02484806-1500525$ & 194.978 & -60.165 & 11.04 & 11.21 & 11.251 & 0.026 & 11.319 & 0.022 & 11.309 & 0.021 & $\ldots$ \\
\hline $44 \ldots \ldots \ldots \ldots \ldots \ldots$ & $02560556-3555579$ & 239.150 & -62.156 & 12.90 & 12.30 & 12.647 & 0.025 & 12.669 & 0.026 & 12.661 & 0.028 & $\ldots$ \\
\hline
\end{tabular}

Notes. - The data are listed as they appear in the 2MASS point-source catalog. Col. (1): Order number. Col. (2): 2MASS name. Cols. (3) and (4): Galactic coordinates,

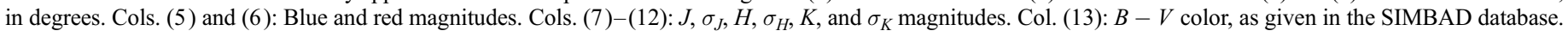

mistaken for A0 stars. In terms of temperature, the values above imply that, for $1 \sigma$ error in the photometry, the minimum temperature in the sample must be $7900 \mathrm{~K}$.

Although both color index restrictions include the $J$ band, the use of two color indices simultaneously gives more reliability to the selection method. Besides, the magnitude criteria stated above also establish an upper limit for distance. This can be estimated assuming that (1) $M_{J}=+0.80$ (for HB stars; Brown et al. 2004), (2) $M_{J} \simeq M_{K}$, (3) there is negligible reddening toward the south Galactic pole in the three infrared bands, and (4) $K \leq 14.3$. Assuming these values, the upper limiting distance of the HB stars turns out to be $5.0 \mathrm{kpc}$. It does not differ much from the lower limit of the Sloan survey, which is near $7 \mathrm{kpc}$, imposed by saturation. Therefore, the Sloan survey and 2MASS complete each other with respect to the magnitude coverage.
Besides using the criteria cited above, due to limitations in the observation time only sources with declination in the range $-56.8^{\circ}<\delta<-13.8^{\circ}$, which corresponds to files aal through abv in the 2MASS database, have been selected. This set amounts to $2182 \mathrm{MASS}$ sources and consists of approximately $80 \%-90 \%$ of the total number of objects with $\delta<-60^{\circ}$. Once this first sample was defined, each object was searched for in the SIMBAD database for information about its nature, such as spectral type, luminosity class, $U B V$ photometry, and counterparts in other catalogs. As mentioned in $\S 1$, other similar surveys have been carried out (Beers et al. 1988, 1992, 1996; Wilhelm et al. 1999a, 1999b; Brown et al. 2003; Sirko et al. 2004), and this study is mainly aimed at stars which have been overlooked in previous surveys. Higher priority is given to objects without any information in the SIMBAD database, especially concerning their spectral 
TABLE 2

Log Book of the Observations

\begin{tabular}{|c|c|c|c|}
\hline Night & Date & Julian Date & $\begin{array}{l}\text { Telescope Size } \\
\text { (m) }\end{array}$ \\
\hline $1 \ldots \ldots \ldots \ldots \ldots .$. & 2003 Oct 25 & $2,452,938$ & 1.60 \\
\hline $2 \ldots \ldots \ldots \ldots \ldots \ldots .$. & 2003 Oct 26 & $2,452,939$ & 1.60 \\
\hline 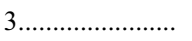 & 2003 Oct 27 & $2,452,940$ & 1.60 \\
\hline 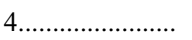 & 2003 Nov 10 & $2,452,954$ & 0.60 \\
\hline $5 \ldots \ldots \ldots \ldots \ldots \ldots \ldots$ & 2003 Nov 11 & $2,452,955$ & 0.60 \\
\hline $6 \ldots \ldots \ldots \ldots \ldots$. & 2004 Sep 4 & $2,453,253$ & 0.60 \\
\hline 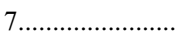 & 2004 Sep 5 & $2,453,254$ & 0.60 \\
\hline 8 & 2004 Sep 6 & $2,453,255$ & 0.60 \\
\hline
\end{tabular}

classification. Forty-four sources fulfilling the criteria above were selected for observation and are listed in Table 1, as they appear in the 2MASS point-source catalog. Since the beginning of the observation runs a few of these stars have been observed and classified by other authors; these cases are noted throughout this paper.

\section{OBSERVATIONS}

Observations were carried out at the Pico dos Dias Observatory, operated by the National Laboratory for Astrophysics (LNA) located in Itajubá, southeast Brazil, during several runs between 2003 and 2005. Two different Boller \& Chivens telescopes were used: a $1.60 \mathrm{~m}$ and a $60 \mathrm{~cm}$, both equipped with the same Cassegrain spectrograph. A long east-west slit of $2^{\prime \prime}$ width was used in all observations. The effective focus ratio was f/13.5 on both tele- scopes, resulting in a reciprocal dispersion of $1.2 \AA$ pixel $^{-1}$ with the 600 line $\mathrm{mm}^{-1}$ grid used.

Table 2 lists the log book of the observations. Image reduction and analysis were performed using the IRAF package, ${ }^{2}$ including the classical procedure to reduce long-slit spectra: bias, dark, and flat-field corrections; spectral profile extraction; and wavelength calibration, which was derived using a He-Ar lamp. After continuum normalization, equivalent widths were calculated assuming Voigt profiles, and a deblending routine was used when necessary. The Voigt profiles were fitted for each diagnostic line using the IRAF splot algorithm. The profile is centered on the line minimum and constrained by the local pseudocontinuum, which is defined by the average values taken from $10 \AA$ wide windows located $25 \AA$ on either side of the line minimum.

\section{RESULTS}

Figure 2 shows the normalized spectra of the 44 stars observed, ordered by right ascension, as displayed in Table 1. Table 3 lists the characteristics of the Balmer series, as well as the $\mathrm{Ca}$ II $\mathrm{K}$ line, according to the definitions proposed by Pier (1983) and Beers et al. (1992) to classify stars among subdwarfs, the HB, degenerate stars, and the MS. The equivalent widths $(W), D_{0.2}, R$, and HP index have all been obtained by fitting a Voigt profile to the lines and are listed in Table 3. Among them, two parameters are more suited to classify the star: the $\mathrm{H} \delta R$-index, which is the

\footnotetext{
${ }^{2}$ IRAF is distributed by the National Optical Astronomy Observatory, which is operated by the Association of Universities for Research in Astronomy, Inc., under contract to the National Science Foundation.
}

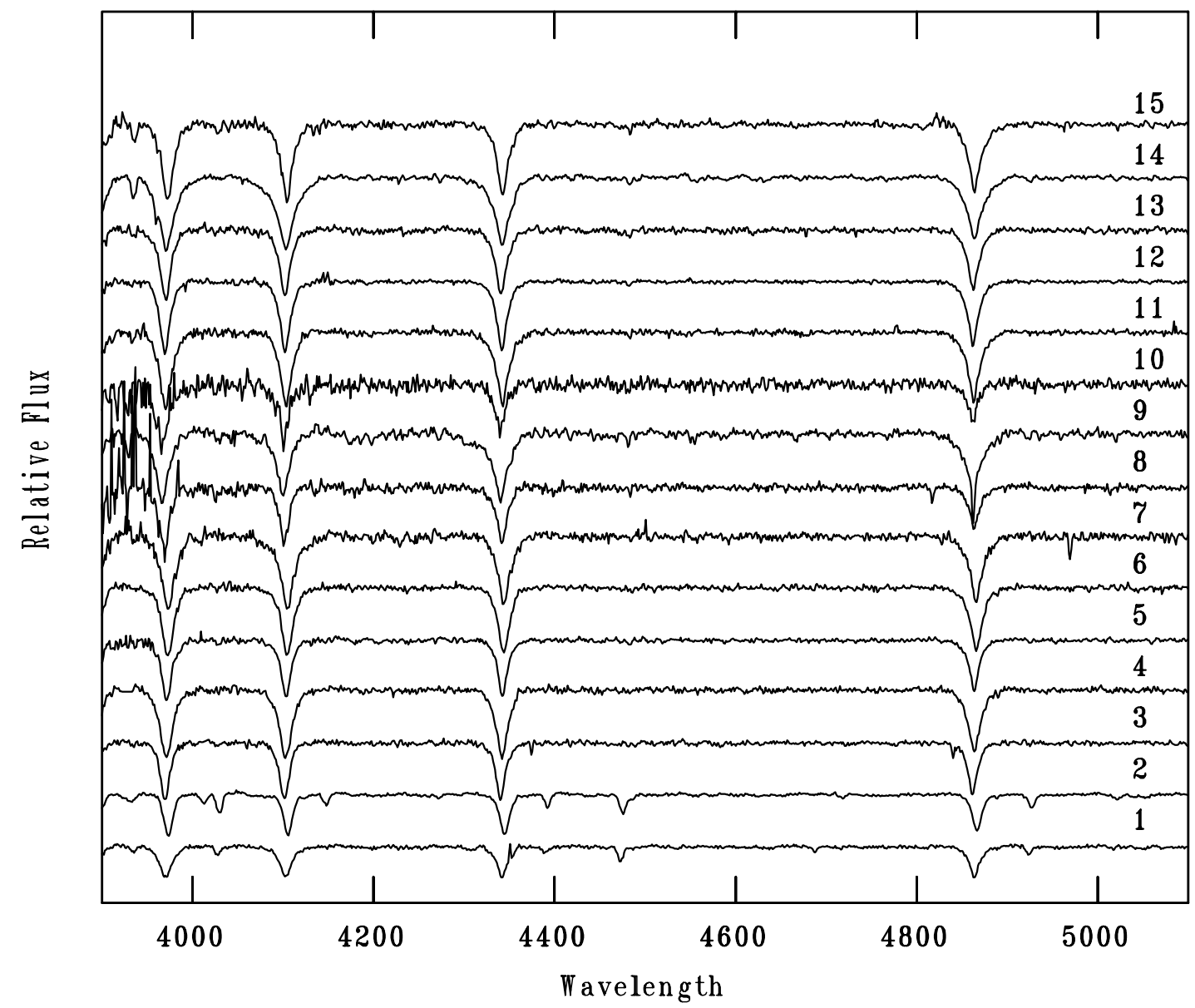

FIG. 2.-Observed spectra of the sample, with their continua normalized. Each number (right-hand side) corresponds to the order given in Table 1. 


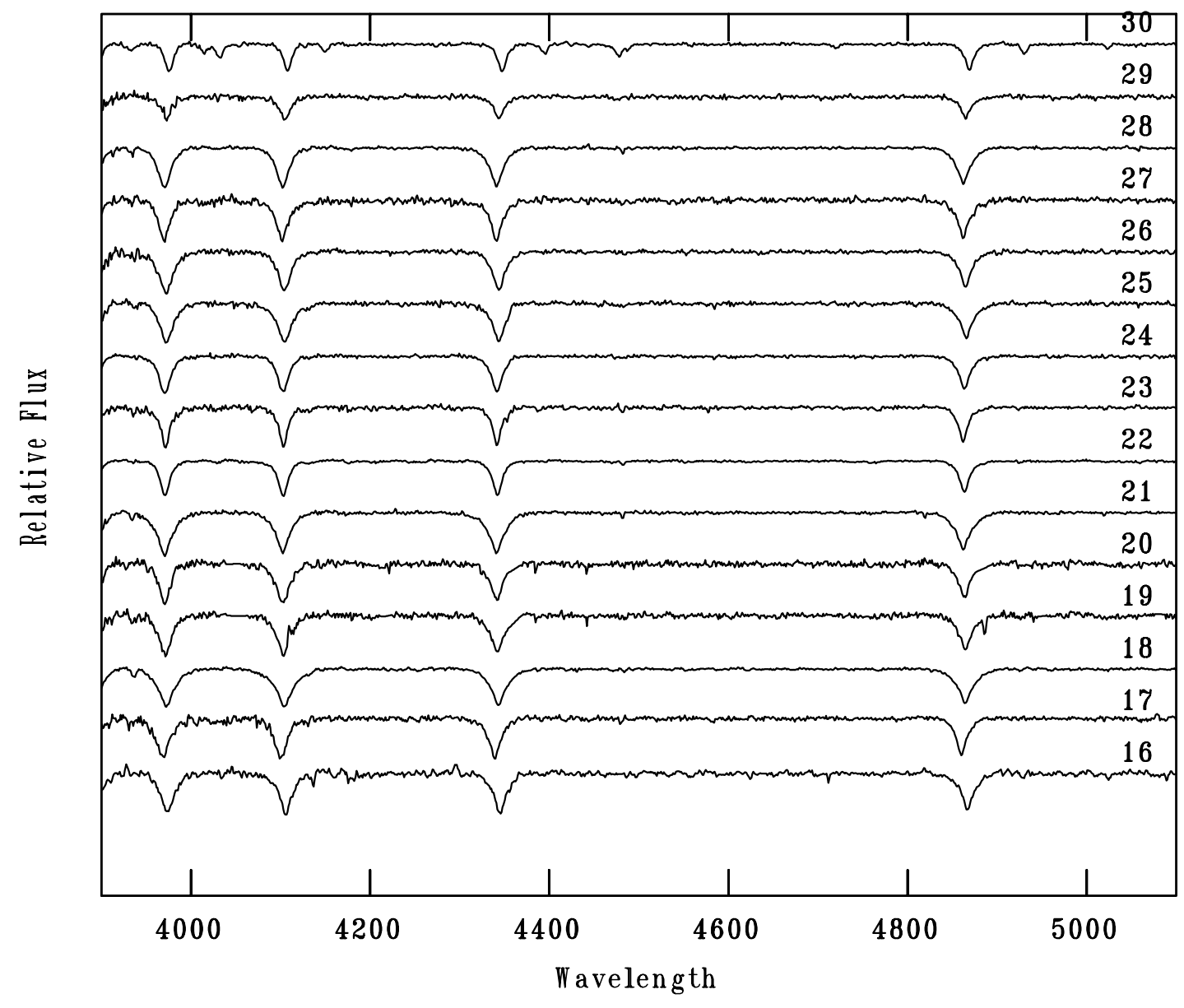

FIG. 2-Continued

ratio between the continuum and the "depth" of the line, and $D_{0.2}$, which is the width of the line taken at 0.8 times the intensity of the continuum (Pier 1983; Wilhelm et al. 1999a). The preference for $\mathrm{H} \delta$ over $\mathrm{H} \gamma$ to perform the calculation of $R$ and $D_{0.2}$ is justified because of the $\mathrm{G}$ band near $\sim 4300 \AA$, which might affect the determination of the continuum near the $\mathrm{H} \gamma$ line, especially among the cooler stars of the sample (Wilhelm et al. 1999a). Stars of each type appear distributed in characteristic regions in the $R$ versus $D_{0.2}$ diagram (Fig. 3 ), although some of the types may appear mixed up, sharing the same portion of the diagram. According to this diagram, five stars belong to the subdwarf category: 22361663-3142130, 22385421-2352209, 01011757-3342454, 02205977-3423353, and 02221984-2324558, whereas all the remaining objects are either A stars or FHB objects. Table 4, which lists the stars with He lines detected, together with the photometric data of Table 1, gives additional information that helps to clarify the nature of these objects. They are discussed in more detail later in this paper. As for the remaining stars, Figure 3 does not allow a reliable classification. The right side of the diagram gathers both the candidate FHB and A stars. As the numerous references in the literature claim (Pier 1983; Sommer-Larsen \& Christensen 1986; Wilhelm et al. 1999a), separating these two classes of objects is difficult because both share the same locus in the $R$ versus $D_{0.2}$ diagram. Nevertheless, there are four objects with $D_{0.2}>35 \AA$, which are far more likely to be A stars.

To disentangle FHB and A stars, the derivation of both the stellar temperature and $\log g$ becomes necessary. The $B-V$ color index is a widely known temperature estimator; however, as Table 1 shows, this information is available for only a few sources. On the other hand, near-infrared photometry is available for all sources, but in spite of several attempts to derive the temperature of hot stars using the $J H K$ color indices (Koornneef 1983; Beers et al. 2007), this method is inaccurate, because the spectral energy distribution approaches the Rayleigh-Jeans regime in the nearinfrared. In this study the temperature and gravity of each star have been determined simultaneously, comparing characteristics of the $\mathrm{H} \beta, \mathrm{H} \gamma$, and $\mathrm{H} \delta$ features with a grid of synthetic spectra calculated by R. L. Kurucz (1993, private communication) assuming solar metallicity and the following ranges of temperature and gravity: $7000 \mathrm{~K} \leq T_{\text {eff }} \leq 12,000 \mathrm{~K}$ and $2.0 \leq \log g \leq 5.0$, with increments of $\Delta T=250 \mathrm{~K}$ for $T_{\text {eff }} \leq 10,000 \mathrm{~K}, \Delta T=500 \mathrm{~K}$ for $T_{\text {eff }}>10,000 \mathrm{~K}$, and $\Delta \log g=0.5$ in all cases. The spectral parameters that were compared with the models are the equivalent widths $(W)$ of $\mathrm{H} \beta, \mathrm{H} \gamma$, and $\mathrm{H} \delta$ and the $D_{0.2}$ of $\mathrm{H} \gamma$ and $\mathrm{H} \delta$. This choice is justified because the Balmer lines are sensitive to both temperature and gravity, which are the main scope of this study. The total $\chi^{2}$ is calculated according to the equation

$$
\begin{aligned}
\chi^{2}= & \frac{\left[W(\mathrm{H} \beta)_{*}-W(\mathrm{H} \beta)_{K}\right]^{2}}{\sigma^{2}(W(\mathrm{H} \beta))}+\frac{\left[W(\mathrm{H} \gamma)_{*}-W(\mathrm{H} \gamma)_{K}\right]^{2}}{\sigma^{2}(W(\mathrm{H} \gamma))} \\
& +\frac{\left[W(\mathrm{H} \delta)_{*}-W(\mathrm{H} \delta)_{K}\right]^{2}}{\sigma^{2}(W(\mathrm{H} \delta))}+\frac{\left[D_{0.2}(\mathrm{H} \gamma)_{*}-D_{0.2}(\mathrm{H} \gamma)_{K}\right]^{2}}{\sigma^{2}\left(D_{0.2}(\mathrm{H} \gamma)\right)} \\
& +\frac{\left[D_{0.2}(\mathrm{H} \delta)_{*}-D_{0.2}(\mathrm{H} \delta)_{K}\right]^{2}}{\sigma^{2}\left(D_{0.2}(\mathrm{H} \delta)\right)}
\end{aligned}
$$

where $\sigma$ is the error bar of the measurement, typically $1 / 10$ of $W$ and $D_{0.2}$. The asterisk and " $K$ " subscripts correspond to observation 


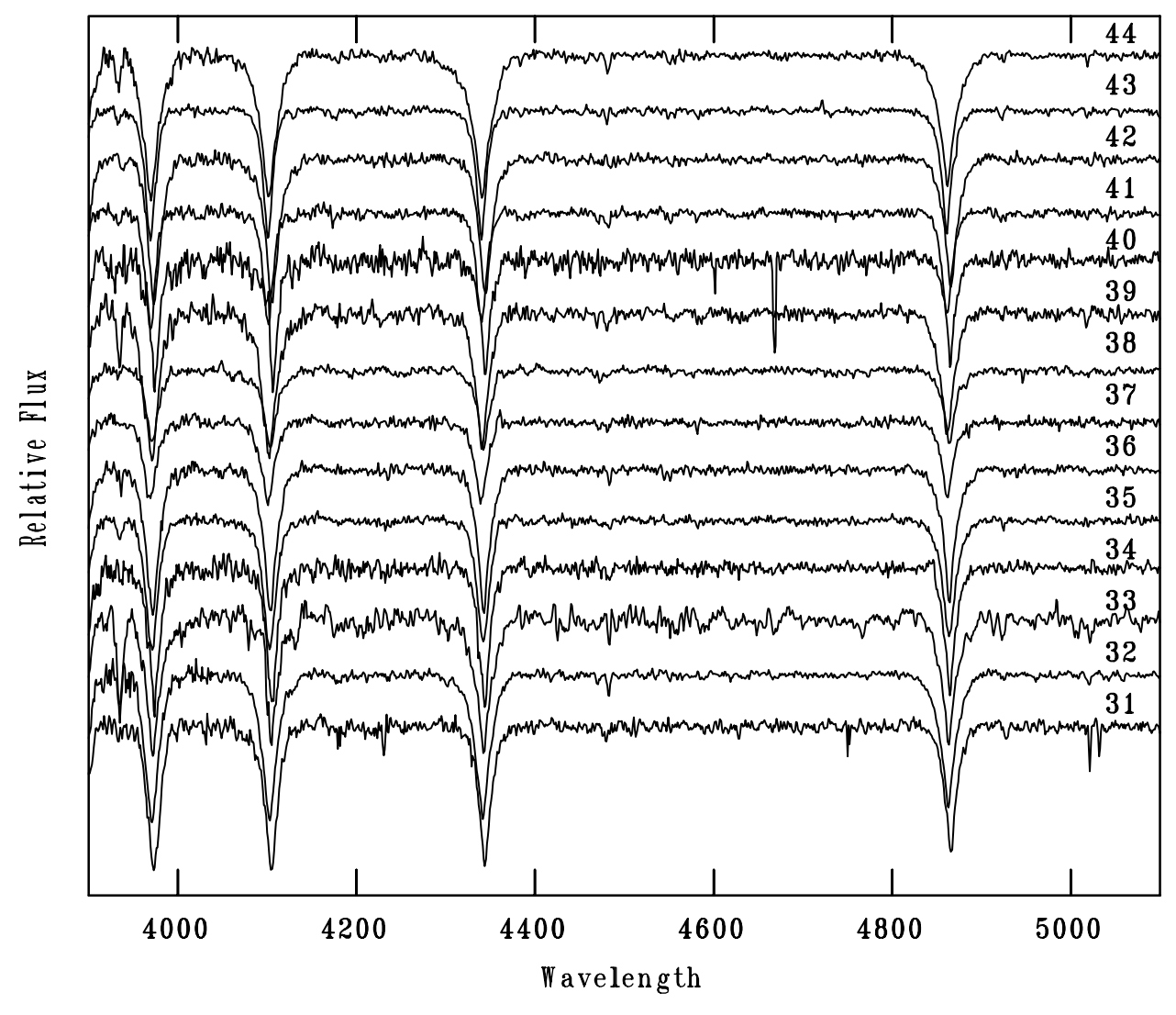

FIG. 2-Continued

and model, respectively. It is well known that the hydrogen lines present a maximum in intensity near the A2 spectral type. As a consequence, for a given value of $\log g$, there are frequently two solutions for $T_{\text {eff }}$, corresponding to the two local minima of $\chi^{2}$. Considering also the range of $\log g$, the number of local minima can be even higher.

We carried out the calculation of $\chi^{2}$ for the whole range of temperature and gravity defined above. In a certain number of cases, when He lines were present or $B-V$ was available, we were able to identify the correct solution among the various local minima. In other cases, when the $\mathrm{Ca}$ II $\mathrm{K}$ line was detected, $W_{K}$ was used to discard local $\chi^{2}$ minima whose temperatures were incompatible with the theoretical models. This was done using the $T_{\text {eff }}$ versus $W_{K}$ diagram proposed by Wilhelm et al. (1999a), calculated for a wide range of temperature and metallicities, which is based on Kurucz's grid of models previously cited in this section. For stars with $W_{K}$ measurements, incorrect $\left(T_{\text {eff }}, \log g\right.$ ) solutions were discarded if their measured $W_{K}$ was incompatible with the synthetic figure corresponding to that temperature (Fig. 2 of Wilhelm et al. 1999a). Nevertheless, even all these techniques together left a considerable number of stars with multiple solutions of $T_{\text {eff }}$ and $\log g$. All single and multiple solutions are presented in Table 5, which includes all sources observed, except those previously identified as subdwarfs according to the diagram depicted in Figure 3.

In order to test the method used for determining the stellar temperatures, we applied it to a test sample, containing stars with temperatures previously determined by a distinct technique. Wilhelm et al. (1999b) determined the $T_{\text {eff }}$ of a sample of FHB stars using a hybrid method that makes use of the $B-V$ and $U-B$ colors, as well as $D_{0.2}$ of $\mathrm{H} \delta$ and $\mathrm{H} \gamma$ (Wilhelm et al. 1999a). According to that method, synthetic values are obtained from the models proposed by R. L. Kurucz (1993, private communication), which are compared with the values obtained from the observations, according to a pondering function that varies with the temperature. Figures $4 a$ and $4 b$ show the stellar temperatures of a subsample of FHB stars (collected from the list published by Wilhelm et al. 1999b) calculated by both methods. A clear bias seems to exist, showing that the present method produces higher temperatures when compared with the results of Wilhelm et al. (1999b). A similar bias was noticed by Kinman \& Castelli (2002), who used $V, J, H$, and $K$ magnitudes to calibrate a gravity- and metallicitydependent relationship between these color indices and $T_{\text {eff }}$. According to that study, the temperatures determined by Wilhelm et al. (1999b) are systematically cooler than those determined from the 2MASS color indices by an amount that increases with $T_{\text {eff }}$.

Since $B-V$ and $E(B-V)$ are both available for practically all the stars in the sample of Wilhelm et al. (1999b), we also compared our method with a well-established relationship between $(B-V)_{0}$ and $T_{\text {eff }}$. This relationship (Gray 1992) was found from observations carried out by various authors who obtained $U B V$ photometry relative to stars in a wide range of gravities and temperatures. We did not use the relationships proposed by Kinman $\&$ Castelli (2002), because a comparison between $B$ and $V$ magnitudes taken from the $2 \mathrm{MASS}$ catalog and the same magnitudes measured by other authors (available in the literature) shows large error bars for magnitudes beyond $B=11.0$.

Figures $4 c$ and $4 d$ show that no large bias seems to exist between the temperatures determined in this work and those obtained using the $(B-V)_{0}$ versus $T_{\text {eff }}$ relationship, within the temperature range $7000 \mathrm{~K}<T_{\text {eff }}<12,000 \mathrm{~K}$. The difference $\Delta T=T_{\text {this work }}-T_{B-V}$ follows a Gaussian distribution centered at $\Delta T=-180 \mathrm{~K}$ and half-width $\sigma=700 \mathrm{~K}$. However, this scattering does not result only from the uncertainty of the present method, but is also due to some scattering present in the $(B-V)_{0}$ versus $T_{\text {eff }}$ relationship. Although Gray (1992) points out that no 
TABLE 3

Characteristics of the Hydrogen Features in the Spectra

\begin{tabular}{|c|c|c|c|c|c|c|c|c|c|c|}
\hline \multirow[b]{2}{*}{$\begin{array}{c}\text { 2MASS NAME } \\
\text { (1) }\end{array}$} & \multicolumn{3}{|c|}{$W$ (VoIGT) $(\AA)$} & \multicolumn{2}{|c|}{$D_{0.2}$} & \multicolumn{2}{|c|}{$R$} & \multirow[b]{2}{*}{$\begin{array}{l}\text { HP INDEX } \\
\text { (9) }\end{array}$} & \multirow{2}{*}{$\begin{array}{c}W \text { (Voigt) Ca II K } \\
(\AA) \\
(10)\end{array}$} & \multirow[b]{2}{*}{$\begin{array}{c}\text { NIGHT } \\
(11)\end{array}$} \\
\hline & $\begin{array}{l}\mathrm{H} \beta \\
(2)\end{array}$ & $\begin{array}{l}\mathrm{H} \gamma \\
(3)\end{array}$ & $\begin{array}{l}\mathrm{H} \delta \\
(4)\end{array}$ & $\begin{array}{l}\mathrm{H} \gamma \\
(5)\end{array}$ & $\begin{array}{l}\mathrm{H} \delta \\
(6)\end{array}$ & $\begin{array}{l}\mathrm{H} \gamma \\
(7)\end{array}$ & $\begin{array}{l}\mathrm{H} \delta \\
(8)\end{array}$ & & & \\
\hline 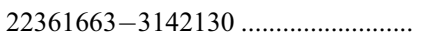 & 6.1 & 5.3 & 6.8 & 8.7 & 10.5 & 0.29 & 0.28 & 6.2 & $\ldots$ & 1 \\
\hline 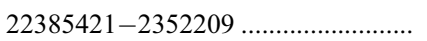 & 6.0 & 6.2 & 5.6 & 11.7 & 11.1 & 0.38 & 0.39 & 6.2 & $\ldots$ & 2 \\
\hline 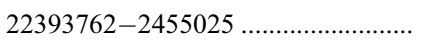 & 9.6 & 10.0 & 10.2 & 15.9 & 18.1 & 0.55 & 0.56 & 10.7 & $\ldots$ & 4 \\
\hline $22475352-3829408$ & 15.1 & 14.2 & 16.0 & 23.3 & 23.5 & 0.67 & 0.66 & 16.1 & $\ldots$ & 1 \\
\hline 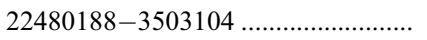 & 9.5 & 10.0 & 13.4 & 15.4 & 19.4 & 0.54 & 0.57 & 12.3 & $\ldots$ & 5 \\
\hline $22501122-3759588 \ldots \ldots \ldots \ldots \ldots \ldots \ldots$ & 14.5 & 12.2 & 14.8 & 21.2 & 22.3 & 0.62 & 0.65 & 14.3 & $\ldots$ & 2 \\
\hline $22515349-4355577$..................................... & 15.9 & 18.0 & 21.6 & 27.7 & 29.1 & 0.67 & 0.71 & 21.1 & $\ldots$ & 5 \\
\hline 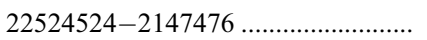 & 8.6 & 12.2 & 12.3 & 20.2 & 20.1 & 0.55 & 0.60 & 13.0 & & 6 \\
\hline 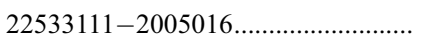 & 19.7 & 17.2 & 23.3 & 26.7 & 33.0 & 0.61 & 0.60 & 21.5 & 1.30 & 6 \\
\hline $22574779-1822567$...................................... & 8.1 & 11.7 & 8.2 & 18.0 & 11.6 & 0.55 & 0.64 & 10.7 & $\ldots$ & 6 \\
\hline $22575321-3250528 \ldots \ldots \ldots \ldots \ldots \ldots \ldots . .$. & 15.9 & 17.9 & 19.7 & 26.4 & 29.6 & 0.73 & 0.75 & 20.1 & 0.50 & 6 \\
\hline 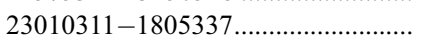 & 12.7 & 13.6 & 14.8 & 20.3 & 23.3 & 0.67 & 0.69 & 15.1 & $\ldots$ & 6 \\
\hline 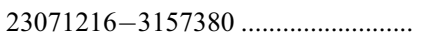 & 12.0 & 14.9 & 17.1 & 23.0 & 25.7 & 0.62 & 0.66 & 17.0 & $\ldots$ & 4 \\
\hline 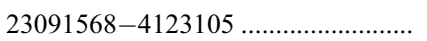 & 20.9 & 19.3 & 24.0 & 30.0 & 36.3 & 0.66 & 0.70 & 23.1 & 1.30 & 3 \\
\hline 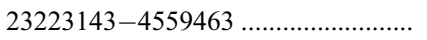 & 14.2 & 13.4 & 16.0 & 20.7 & 23.3 & 0.68 & 0.76 & 15.6 & 0.89 & 7 \\
\hline $23270592-4009350$ & 17.4 & 19.8 & 24.8 & 31.4 & 34.8 & 0.71 & 0.75 & 23.8 & $\ldots$ & 4 \\
\hline 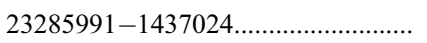 & 14.0 & 16.3 & 10.1 & 23.6 & 19.2 & 0.70 & 0.66 & 14.4 & $\ldots$ & 7 \\
\hline 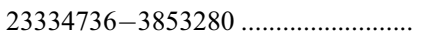 & 19.9 & 20.5 & 25.4 & 32.2 & 38.0 & 0.64 & 0.67 & 24.5 & 0.96 & 5 \\
\hline $23462825-3455590$ & 15.5 & 17.6 & 15.2 & 26.7 & 19.8 & 0.62 & 0.70 & 17.7 & $\ldots$ & 3 \\
\hline 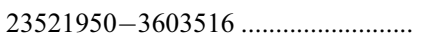 & 14.6 & 15.2 & 16.9 & 23.2 & 25.6 & 0.62 & 0.66 & 17.1 & $\ldots$ & 3 \\
\hline 23591014-4342306 ……...................... & 20.0 & 21.3 & 25.5 & 32.7 & 38.0 & 0.72 & 0.74 & 25.0 & 0.51 & 8 \\
\hline 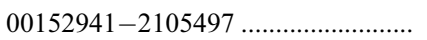 & 10.9 & 10.7 & 11.0 & 17.6 & 17.2 & 0.59 & 0.60 & 11.5 & $\ldots$ & 3 \\
\hline 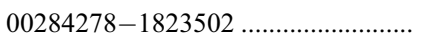 & 11.3 & 10.9 & 9.7 & 15.6 & 16.6 & 0.65 & 0.67 & 11.0 & 0.46 & 7 \\
\hline 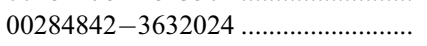 & 12.7 & 12.8 & 13.6 & 20.6 & 21.5 & 0.61 & 0.62 & 14.1 & $\ldots$ & 2 \\
\hline 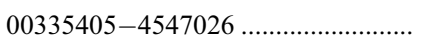 & 16.1 & 14.6 & 19.4 & 24.3 & 27.5 & 0.66 & 0.65 & 18.0 & $\ldots$ & 2 \\
\hline 00383181-3913183 ………................. & 16.1 & 17.1 & 22.2 & 26.5 & 29.7 & 0.68 & 0.70 & 20.9 & $\ldots$ & 4 \\
\hline 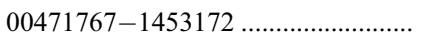 & 15.4 & 16.1 & 17.0 & 24.4 & 22.9 & 0.72 & 0.73 & 17.7 & $\ldots$ & 8 \\
\hline 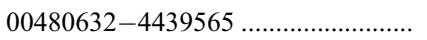 & 17.0 & 18.2 & 20.5 & 26.3 & 29.8 & 0.69 & 0.72 & 20.7 & 0.24 & 8 \\
\hline 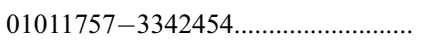 & 8.3 & 9.5 & 13.3 & 14.0 & 18.6 & 0.39 & 0.45 & 12.0 & $\ldots$ & 4 \\
\hline 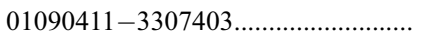 & 7.8 & 7.6 & 8.6 & 16.0 & 13.2 & 0.44 & 0.67 & 8.5 & $\ldots$ & 5 \\
\hline 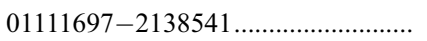 & 14.9 & 15.8 & 17.7 & 30.5 & 23.3 & 0.68 & 0.72 & 17.9 & $\ldots$ & 5 \\
\hline 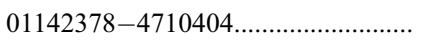 & 20.3 & 21.7 & 23.4 & 32.7 & 33.7 & 0.71 & 0.73 & 24.2 & 0.72 & 8 \\
\hline 01294009-4015539 ……...................... & 17.0 & 17.4 & $\ldots$ & 29.7 & $\ldots$ & 0.65 & $\ldots$ & 59.9 & 4.50 & 7 \\
\hline 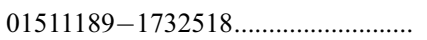 & 12.3 & 13.0 & 9.1 & 19.6 & 16.5 & 0.68 & 0.63 & 11.9 & $\ldots$ & 7 \\
\hline 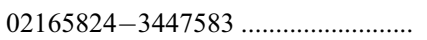 & 14.7 & 12.3 & 16.9 & 21.9 & 24.9 & 0.59 & 0.63 & 15.4 & 0.53 & 2 \\
\hline 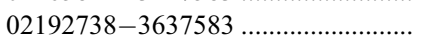 & 16.1 & 17.6 & 21.2 & 27.8 & 30.4 & 0.71 & 0.71 & 20.7 & 0.31 & 8 \\
\hline 02205977-3423353 …………............. & 8.9 & 8.1 & 10.8 & 15.1 & 17.8 & 0.39 & 0.41 & 9.9 & $\ldots$ & 2 \\
\hline 02221984-2324558 …….................... & 8.4 & 8.1 & 9.8 & 13.5 & 16.2 & 0.39 & 0.38 & 9.4 & $\ldots$ & 3 \\
\hline 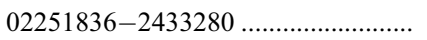 & 15.7 & 18.7 & 22.3 & 28.7 & 31.9 & 0.68 & 0.72 & 21.9 & 1.23 & 4 \\
\hline 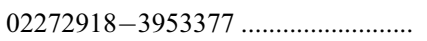 & 9.1 & 10.1 & 6.1 & 16.4 & 10.1 & 0.56 & 0.59 & 8.7 & $\ldots$ & 7 \\
\hline 02324780-2038431 …….................... & 10.2 & 9.4 & 10.1 & 15.9 & 16.8 & 0.53 & 0.55 & 10.3 & $\ldots$ & 2 \\
\hline 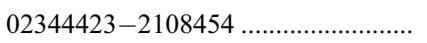 & 15.2 & 18.1 & 21.5 & 26.3 & 30.4 & 0.67 & 0.71 & 21.1 & $\ldots$ & 4 \\
\hline 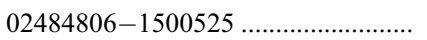 & 12.8 & 12.7 & 13.9 & 19.6 & 21.1 & 0.64 & 0.64 & 14.1 & 0.39 & 8 \\
\hline 02560556-3555579 ……..................... & 21.2 & 23.9 & 27.0 & 36.6 & 39.6 & 0.70 & 0.72 & 27.3 & 1.08 & 8 \\
\hline
\end{tabular}

Notes.-Col. (1): Name of the 2MASS source. Cols. (2)-(4): Voigt equivalent widths of the $\mathrm{H} \beta, \mathrm{H} \gamma$, and $\mathrm{H} \delta$ lines, respectively. Cols. (5) and (6): $D_{0.2}$ of the $\mathrm{H} \gamma$ and $\mathrm{H} \delta$ lines, respectively. Cols. (7) and (8): $R$ of $\mathrm{H} \gamma$ and $\mathrm{H} \delta$, respectively. Col. (9): HP index, defined by Pier (1983), Sommer-Larsen \& Christensen (1986), and Wilhelm et al. (1999a). Col. (10): Voigt equivalent width of the Ca II K line. Col. (11): Night number (see Table 2).

clear dependence seems to exist on $\log g$ or $[\mathrm{Fe} / \mathrm{H}]$, an intrinsic scattering resulting from the various observational conditions is present. This scattering varies with $(B-V)_{0}$, being typically $2 \%-3 \%$ of the temperature for an increment $\sigma(B-V)_{0}=0.01$, but it might be as high as $4 \%$ if $(B-V)_{0}<0.0$. In fact, a good determination of the dereddened $(B-V)_{0}$ requires, besides good photometric observations, an accurate estimation of the color excess $E(B-V)$. These two effects combined, and assuming $\sigma(B-$ $V)_{0}=0.01$, imply an uncertainty in temperature of $200 \mathrm{~K}$ at $T_{\text {eff }}=7000 \mathrm{~K}$ or $440 \mathrm{~K}$ for $T_{\text {eff }}=12,000 \mathrm{~K}$. Part of the width of the $\Delta T$ distribution observed in Figure $4 d$ (half-width $\sigma=700 \mathrm{~K}$ ) is due to this effect, whereas another part is caused by the error of the present method only. Thus, we conclude that the intrinsic error bar of the method for determining $T_{\text {eff }}$ proposed in this work, which is not based on $(B-V)_{0}$ but on some features of the Balmer series, is about $500 \mathrm{~K}$, on average.

Figure 5 illustrates a well-known tool proposed by Wilhelm et al. (1999a) to visualize the stellar parameters $T_{\text {eff }}$ and $\log g$. The input data to this plot are given in Table 5 . The uniquely determined solutions of $T_{\text {eff }}$ and $\log g$ are shown as filled circles, whereas the multiple solutions are shown as open circles. An examination of Figure 5 reveals that the stars in the sample are distributed over a wide range of gravities, $2.0 \leq \log g \leq 5$.0. This excludes low-gravity objects such as post-AGB stars, which are 


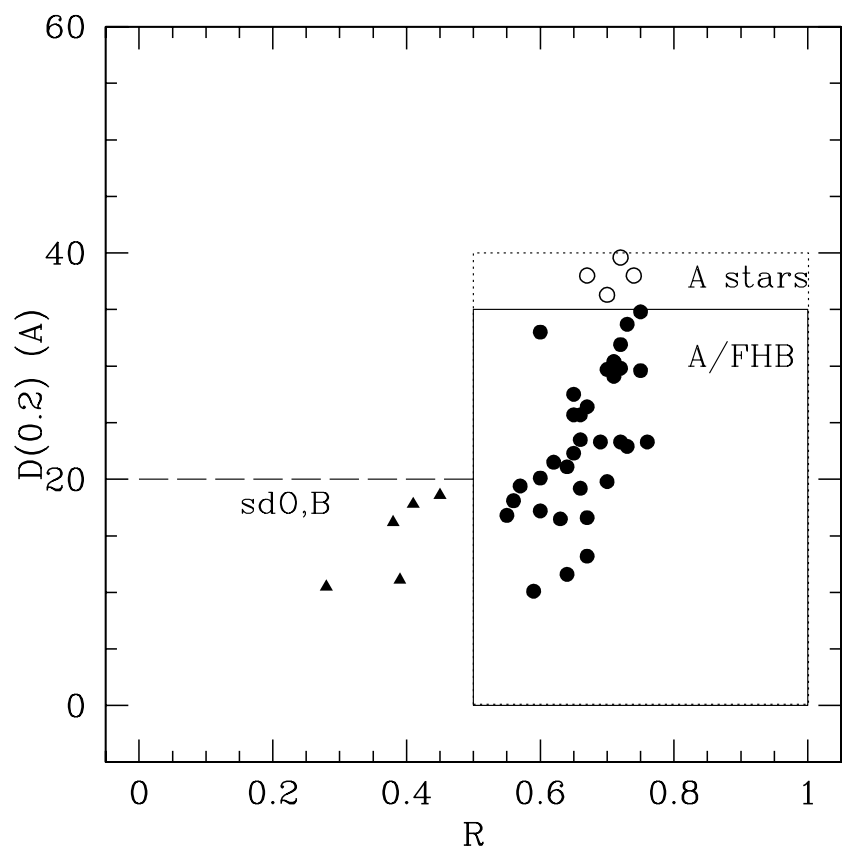

FIG. 3.-Sample in $R$ vs. $\mathrm{H} \delta D_{0.2}$, calculated using the $\mathrm{H} \delta$ line as given in Table 3. The symbols used in this preliminary classification are as follows: sdO, $\mathrm{sdB}$ (triangles), A/FHB stars ( filled circles), and MS A stars (open circles).

often discovered at high latitudes, as in this survey. The range of $\log g$ values also excludes degenerate stars such as white dwarfs. On the other hand, the range of $\log g$ does include both FHB and A stars and, in a limited number of cases, allows discrimination between these two categories. This especially applies to the objects located on the right side of the diagram, where $T_{\text {eff }}<10,000 \mathrm{~K}$, and the four objects in the upper box of Figure 3.

Unfortunately, because the sample was mainly aimed at objects overlooked in previous surveys, no data on radial velocity exist in the literature, except in the cases of 23462825-3455590 and 23521950-3603516 (Wilhelm et al. 1999b).

\subsection{Subdwarfs}

As noted above, five stars are classified as subdwarfs according to the $R$ versus $D_{0.2}$ diagram. The star 22361663-3142130 was previously identified as a subdwarf (Kilkenny et al. 1988). The several He I and He II lines present in its spectrum (Table 4) and its $B-V$ color index (Table 1) support its classification as an sdO star. The star 22385421-2352209 has numerous He I lines, but $\mathrm{He}$ II is absent. Its $B-V$ color is also consistent with its classification as an sdB star. The star 01011757-3342454 does not show He lines in the present spectrum, perhaps because of its low $\mathrm{S} / \mathrm{N}$. Nevertheless, this object is a well-known sdB star showing high radial velocity (Bergeron et al. 1984; Altmann et al. 2004; Edelmann et al. 2005). Its position in the $R$ versus $D_{0.2}$ diagram only confirms its previous classification. The spectrum of 02221984-2324558 exhibits the He I $\lambda 4471$ line, suggesting its classification as $\mathrm{sdB}$. It has been recently cataloged as a subdwarf B binary in the Edinburgh-Cape survey (Morales-Rueda et al. 2006). Like 02221984-2324558, the star 02205977-3423353 was only recently classified as sdB (Altmann et al. 2004), after the beginning of the present survey. Table 1 tells us that it is one of the faintest objects in the sample. Its He I lines remain undetected, probably because of the low $\mathrm{S} / \mathrm{N}$ and the fact that the Balmer lines in subdwarfs are typically shallow.

\subsection{A-Type Stars}

Figure 3 shows four stars that appear in the region of the $R$ versus $D_{0.2}$ diagram where only A-type stars are commonly found. None of them have been previously confirmed as A stars. These objects are 23091568-4123105, 23334736-3853280, 235910144342206, and 02560556-3555579. Table 5 shows $\log g \geq 4.0$ for all of them, confirming this classification. The measurement of the $\mathrm{Ca}$ II $\mathrm{K}$ line helped to remove the degeneracy of the multiple solutions of $T_{\text {eff }}$ of the following stars: 23091568-4123105, 23591014-4342206, and 23334736-3853280. According to the theoretical models, the strength of the $\mathrm{Ca}$ II $\mathrm{K}$ line indicates that the former two objects have subsolar $[\mathrm{Fe} / \mathrm{H}]$, whereas the latter probably has nearly solar metallicity.

Besides the A stars clearly classified using the $R$ versus $D_{0.2}$ diagram, there are several borderline cases showing $\mathrm{H} \delta D_{0.2}$ in the range $30-35 \AA$. The stars belonging to this category are 22533111-2005016, 23270592-4009350, 01142378-4710404, 02192738-3637583, 02251836-2433280, and 023444232108454. The objects 22533111-2005016 and 022518362433280 both exhibit strong Ca II K lines, which discards the $\chi^{2}$ high-temperature solution, confirming them as A-type stars. The theoretical models indicate that $01142378-4710404$ and 02192738-3637583 must be low-metallicity stars. Considering what has been exposed above, one can state that the former three objects are confirmed A stars, whereas the latter three are in fact borderline cases, because their $\chi^{2}$ solutions are compatible with both A and FHB classifications. Among them, only 02344423-2108454 has been previously observed in some detail (Kilkenny \& Muller 1989; Kilkenny 1995). There are no $\mathrm{H} \delta$ measurements for the star 01294009-4015539 which could allow its classification using the method applied to the other stars in the sample. However, its $\mathrm{H} \gamma$ line characteristics suggest that it might be either an A or a FHB star. On the other hand, the high intensity of the $\mathrm{Ca}$ II $\mathrm{K}$ line suggests that its metallicity is near solar,

TABLE 4

Equivalent Widths $(\AA ̊)$ of the Helium Lines Detected in the Survey

\begin{tabular}{|c|c|c|c|c|c|c|c|}
\hline 2MASS Name & Не г $\lambda 4009$ & He I $\lambda 4026$ & He I $\lambda 4144$ & He I $\lambda 4388$ & He I $\lambda 4471$ & Hе г $\lambda 4922$ & He II $\lambda 4686$ \\
\hline $22361663-3142130 \ldots \ldots \ldots \ldots \ldots$ & . & 0.61 & $\ldots$ & 0.41 & 0.98 & 0.59 & 0.34 \\
\hline $22385421-2352209$.................... & 0.86 & 1.51 & 0.81 & 0.99 & 2.01 & 1.18 & $\ldots$ \\
\hline 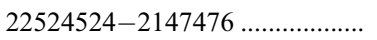 & $\ldots$ & 0.32 & $\ldots$ & $\ldots$ & $\ldots$ & $\ldots$ & $\ldots$ \\
\hline $01090411-3307403 \ldots \ldots \ldots \ldots \ldots \ldots$ & 1.81 & 2.15 & 1.11 & 1.65 & 2.98 & 1.36 & $\ldots$ \\
\hline 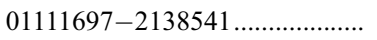 & $\ldots$ & $\ldots$ & $\ldots$ & $\ldots$ & $\ldots$ & 0.51 & $\ldots$ \\
\hline 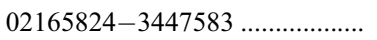 & $\ldots$ & $\ldots$ & $\ldots$ & $\ldots$ & $\ldots$ & 0.12 & $\ldots$ \\
\hline 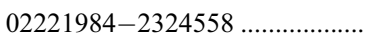 & $\ldots$ & $\ldots$ & $\ldots$ & $\ldots$ & 0.16 & $\ldots$ & $\ldots$ \\
\hline 02324780-2038431 ……............ & $\ldots$ & $\ldots$ & $\ldots$ & $\ldots$ & 0.18 & $\ldots$ & $\ldots$ \\
\hline
\end{tabular}

NotE.-All lines have been fitted using a Voigt profile. 
TABLE 5

Stellar Temperature and Gravity of the Objects Surveyed

\begin{tabular}{|c|c|c|c|c|c|c|c|c|c|c|c|}
\hline 2MASS Name & $\begin{array}{c}\mathrm{H} \delta D_{0.2} \\
(\AA)\end{array}$ & $\begin{array}{c}T_{1} \\
(\mathrm{~K})\end{array}$ & $\log g$ & $\chi_{1}^{2}$ & $\begin{array}{c}T_{2} \\
(\mathrm{~K})\end{array}$ & $\log g$ & $\chi_{2}^{2}$ & $\begin{array}{c}T_{3} \\
(\mathrm{~K})\end{array}$ & $\log g$ & $\chi_{3}^{2}$ & Class \\
\hline $22393762-2455025 \ldots \ldots \ldots \ldots$ & 18.1 & $>17000$ & 4.5 & 2.7 & & $\ldots$ & $\ldots$ & $\ldots$ & $\ldots$ & $\ldots$ & A \\
\hline $22475352-3829408 \ldots \ldots \ldots \ldots$ & 23.5 & 7750 & 3.0 & 1.8 & 12500 & 4.5 & 0.7 & $\ldots$ & $\ldots$ & $\ldots$ & $\ldots$ \\
\hline $22480188-3503104 \ldots \ldots \ldots \ldots$ & 19.4 & 7500 & 4.5 & 15.7 & 10000 & 3.0 & 15.4 & 11500 & 3.5 & 11.4 & $\ldots$ \\
\hline $22501122-3759588 \ldots \ldots \ldots \ldots$ & 22.3 & 7500 & 3.0 & 5.2 & 10000 & 3.5 & 5.2 & 11500 & 4.0 & 3.5 & $\ldots$ \\
\hline $22515349-4355577 \ldots \ldots \ldots \ldots$ & 29.1 & 8250 & 3.5 & 3.8 & 11000 & 4.5 & 4.0 & $\ldots$ & $\ldots$ & $\ldots$ & $\ldots$ \\
\hline $22524524-2147476 \ldots \ldots \ldots \ldots$ & 20.1 & 11500 & 3.5 & 21.1 & 13000 & 4.0 & 15.5 & 16000 & 4.5 & 14.3 & $\ldots$ \\
\hline $22533111-2005016 \ldots \ldots \ldots \ldots$ & 33.0 & 8250 & 4.0 & 6.9 & $\ldots$ & $\ldots$ & $\ldots$ & $\ldots$ & $\ldots$ & $\ldots$ & A \\
\hline $22574779-1822567 \ldots \ldots \ldots . .$. & 11.6 & 7000 & 3.5 & 18.6 & 11000 & 3.0 & 18.1 & 13000 & 3.5 & 15.1 & $\ldots$ \\
\hline $22575321-3250528^{\mathrm{a}} \ldots \ldots \ldots \ldots$ & 29.6 & 8250 & 3.5 & 2.8 & $\ldots$ & $\ldots$ & $\ldots$ & $\ldots$ & $\ldots$ & $\ldots$ & FHB \\
\hline $23010311-1805337 \ldots \ldots \ldots \ldots . . .$. & 23.3 & 7500 & 4.0 & 6.6 & 10000 & 3.5 & 5.0 & 13000 & 4.5 & 2.0 & $\ldots$ \\
\hline $23071216-3157380 \ldots \ldots \ldots . .$. & 25.7 & 7750 & 3.0 & 12.1 & 8750 & 3.0 & 14.2 & 10000 & 3.5 & 12.8 & $\ldots$ \\
\hline $23091568-4123105 \ldots \ldots \ldots \ldots$ & 36.3 & 8500 & 4.0 & 3.1 & $\ldots$ & $\ldots$ & $\ldots$ & $\ldots$ & $\ldots$ & $\ldots$ & $\mathrm{A}$ \\
\hline $23223143-4559463 \ldots \ldots \ldots . .$. & 23.3 & 10000 & 3.5 & 3.7 & $\ldots$ & $\ldots$ & $\ldots$ & $\ldots$ & $\ldots$ & $\ldots$ & $\ldots$ \\
\hline $23270592-4009350 \ldots \ldots \ldots . .$. & 34.8 & 8500 & 4.0 & 4.0 & $\ldots$ & $\ldots$ & $\ldots$ & $\ldots$ & $\ldots$ & $\ldots$ & A \\
\hline 23285991-1437024............. & 19.2 & 7500 & 4.0 & 13.6 & 9250 & 3.0 & 16.0 & $\ldots$ & $\ldots$ & $\ldots$ & $\ldots$ \\
\hline $23334736-3853280 \ldots \ldots \ldots . .$. & 38.0 & 9500 & 4.5 & 3.6 & $\ldots$ & $\ldots$ & $\ldots$ & $\ldots$ & $\ldots$ & $\ldots$ & A \\
\hline $23462825-3455590 \ldots \ldots \ldots . .$. & 19.8 & 8750 & 3.0 & 3.9 & $\ldots$ & $\ldots$ & $\ldots$ & $\ldots$ & $\ldots$ & $\ldots$ & FHB \\
\hline $23521950-3603516 \ldots \ldots \ldots \ldots$ & 25.6 & 7750 & 3.0 & 2.5 & 12000 & 4.5 & 1.4 & $\ldots$ & $\ldots$ & $\ldots$ & $\ldots$ \\
\hline 23591014-4342306 ............. & 38.0 & 9500 & 4.5 & 2.5 & $\ldots$ & $\ldots$ & $\ldots$ & $\ldots$ & $\ldots$ & $\ldots$ & $\mathrm{A}$ \\
\hline $00152941-2105497 \ldots \ldots \ldots . .$. & 17.2 & 7500 & 4.5 & 2.2 & 11500 & 3.5 & 2.7 & 13000 & 4.0 & 1.6 & $\ldots$ \\
\hline $00284278-1823502 \ldots \ldots \ldots . .$. & 16.6 & 7000 & 3.0 & 3.0 & 7500 & 4.5 & 2.8 & 10000 & 3.0 & 2.8 & $\ldots$ \\
\hline $00284842-3632024 \ldots \ldots \ldots \ldots$ & 21.5 & 7500 & 4.0 & 4.4 & 14000 & 4.5 & 1.1 & $\ldots$ & $\ldots$ & $\ldots$ & $\ldots$ \\
\hline $00335405-4547026 \ldots \ldots \ldots . .$. & 27.5 & 8250 & 3.0 & 5.8 & 12000 & 4.5 & 4.5 & $\ldots$ & $\ldots$ & $\ldots$ & $\ldots$ \\
\hline $00383181-3913183 \ldots \ldots \ldots . .$. & 29.7 & 8250 & 3.5 & 5.2 & 10000 & 4.0 & 5.4 & $\ldots$ & $\ldots$ & $\ldots$ & $\ldots$ \\
\hline $00471767-1453172 \ldots \ldots \ldots . .$. & 22.9 & 7750 & 3.0 & 0.7 & 8500 & 3.0 & 0.7 & 12000 & 4.5 & 0.6 & $\ldots$ \\
\hline $00480632-4439565^{\mathrm{a}} \ldots \ldots \ldots . .$. & 29.8 & 8250 & 3.5 & 1.8 & 11000 & 4.5 & 2.0 & $\ldots$ & $\ldots$ & $\ldots$ & $\ldots$ \\
\hline $01090411-3307403 \ldots \ldots \ldots \ldots . . .$. & 13.2 & 11500 & 3.0 & 11.1 & $\ldots$ & $\ldots$ & $\ldots$ & $\ldots$ & $\ldots$ & $\ldots$ & FHB \\
\hline $01111697-2138541 \ldots \ldots \ldots \ldots$ & 23.3 & 10500 & 4.0 & 5.8 & 12000 & 4.5 & 5.5 & $\ldots$ & $\ldots$ & $\ldots$ & $\ldots$ \\
\hline $01142378-4710404 \ldots \ldots \ldots \ldots$ & 33.7 & 8750 & 4.5 & 0.7 & 10000 & 4.5 & 0.5 & $\ldots$ & $\ldots$ & $\ldots$ & $\mathrm{A}$ \\
\hline $01511189-1732518 \ldots \ldots \ldots \ldots$ & 16.5 & 7500 & 4.5 & 7.8 & $\ldots$ & $\ldots$ & $\ldots$ & $\ldots$ & $\ldots$ & $\ldots$ & $\mathrm{A}$ \\
\hline $02165824-3447583 \ldots \ldots \ldots . .$. & 24.9 & 10000 & 3.5 & 9.7 & $\ldots$ & $\ldots$ & $\ldots$ & $\ldots$ & $\ldots$ & $\ldots$ & $\ldots$ \\
\hline $02192738-3637583 \ldots \ldots \ldots . .$. & 30.4 & 8250 & 3.5 & 3.8 & 11000 & 4.5 & 3.8 & $\ldots$ & $\ldots$ & $\ldots$ & A \\
\hline $02251836-2433280 \ldots \ldots \ldots . . .$. & 31.9 & 8500 & 3.5 & 6.4 & $\ldots$ & $\ldots$ & $\ldots$ & $\ldots$ & $\ldots$ & $\ldots$ & $\mathrm{A}$ ? \\
\hline $02272918-3953377 \ldots \ldots \ldots . .$. & 10.1 & 7000 & 4.5 & 16.4 & $\ldots$ & $\ldots$ & $\ldots$ & $\ldots$ & $\ldots$ & $\ldots$ & A \\
\hline $02324780-2038431 \ldots \ldots \ldots . .$. & 16.8 & 12000 & 3.5 & 3.8 & 14000 & 4.0 & 2.8 & 17000 & 4.5 & 1.3 & $\ldots$ \\
\hline 02344423-2108454 ............ & 30.4 & 10000 & 4.0 & 6.5 & $\ldots$ & $\ldots$ & $\ldots$ & $\ldots$ & $\ldots$ & $\ldots$ & A \\
\hline $02484806-1500525 \ldots \ldots \ldots . .$. & 21.1 & 7500 & 4.0 & 4.3 & 9250 & 3.0 & 4.2 & 10500 & 3.5 & 3.1 & $\ldots$ \\
\hline $02560556-3555579 \ldots \ldots \ldots . .$. & 39.6 & 9250 & 4.5 & 2.1 & $\ldots$ & $\ldots$ & $\ldots$ & $\ldots$ & $\ldots$ & $\ldots$ & A \\
\hline
\end{tabular}

Notes.-Subdwarfs excluded. Temperatures $T_{1}, T_{2}$, and $T_{3}$ correspond to the various least- $\chi^{2}$ values calculated using eq. (1). The error bars of $T$ and $\log g$ are $500 \mathrm{~K}$ and 0.5 dex, respectively.

${ }^{\text {a }}$ Considering the strength of the $\mathrm{Ca}$ II $\mathrm{K}$ line and the assumption of $T_{\text {eff }}=8250 \mathrm{~K}$ and $\log g=3.5$, this must be a low-metallicity star. 

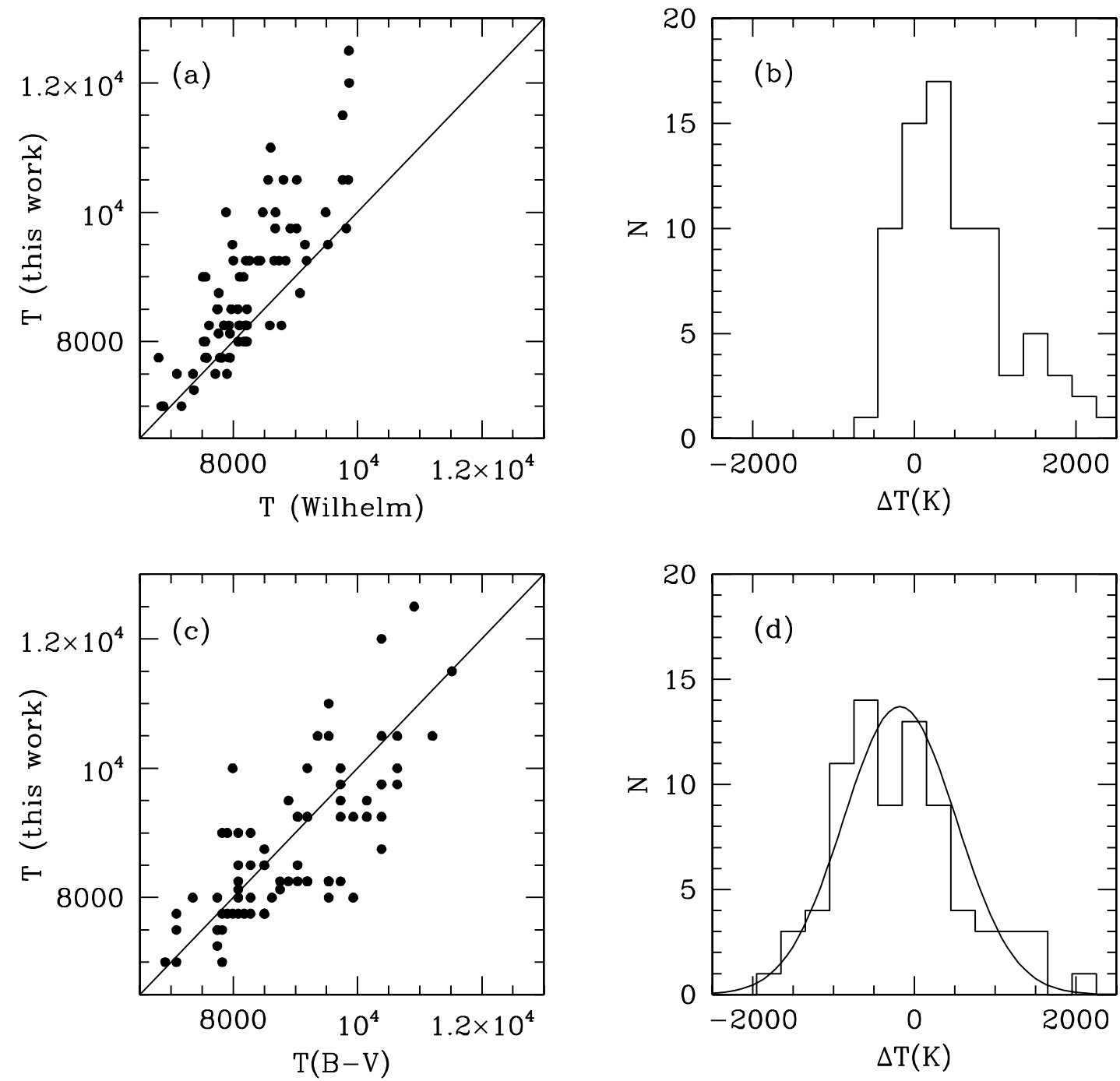

FIG. 4.- $(a, b)$ Comparison between the stellar $T_{\text {eff }}$ determined in this work and by Wilhelm et al. (1999b). $(c, d)$ Same as $(a)$ and $(b)$, but using the $(B-V)_{0}$ vs. $T_{\text {eff }}$ relationship by Gray (1992). In both cases $\Delta T$ represents the difference between the temperature calculated in the present work (based on Kurucz's models) and the other method. 


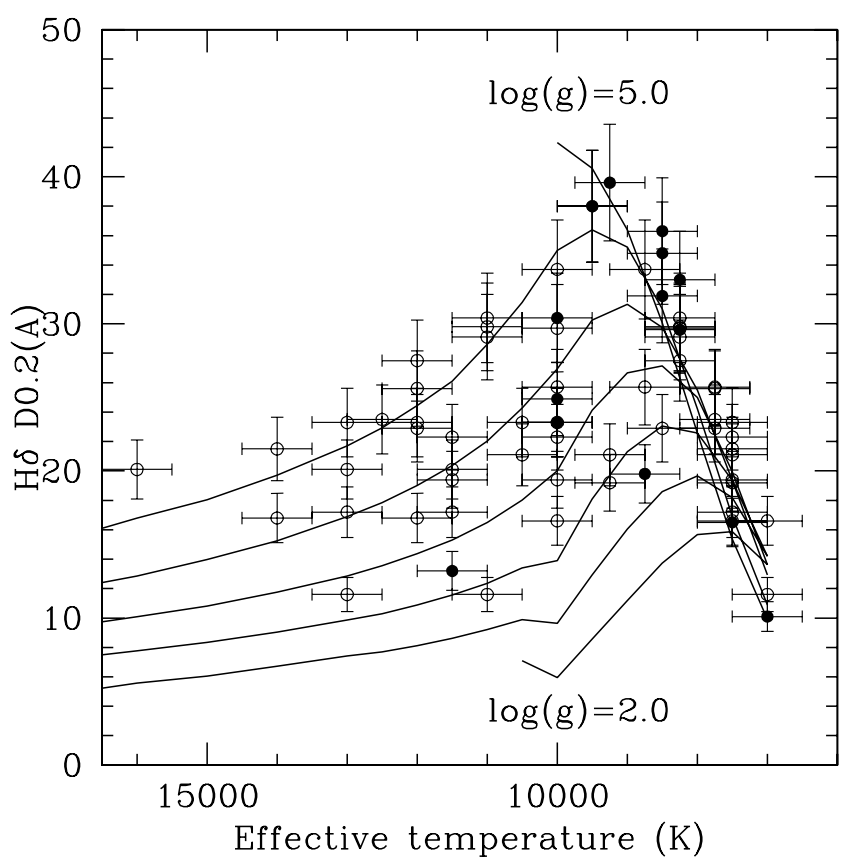

Fig. 5.-Plot of $T_{\text {eff }}$ vs. $\mathrm{H} \delta D_{0.2}$ ( $\AA$ ). Surface gravity and temperature correspond to the least- $\chi^{2}$ values calculated using eq. (1) and given in Table 5. Filled circles represent the unique solutions of $T_{\text {eff }}$ and $\log g$, whereas open circles represent all the remaining multiple solutions. The lines represent the model atmospheres by Kurucz for various $\log g$ values and solar metallicity. Stars identified as subdwarfs in Fig. 3 have been omitted.

which favors the A-type classification. Other stars in Table 5 likely to be A types have been classified according to their $\log g$ values.

The JHK magnitudes can be used to calculate the distance to the Galactic plane of these stars. Assuming the infrared color indices for the MS given by Koornneef (1983), all objects reliably classified as A type are in the range $0.7 \mathrm{kpc}<|z|<2.3 \mathrm{kpc}$ and therefore immersed in the thick disk traced by Gilmore \& Reid (1983). According to that study, the thick-disk population dominates over the thin disk beyond $|z|>1.5 \mathrm{kpc}$, which corresponds to the average $|z|$ value of the A-type stars of the present sample.

\subsection{FHB Stars}

FHB stars frequently appear mixed with A-type stars, especially beyond $T_{\text {eff }}=10,000 \mathrm{~K}$. Disentangling these two kinds of objects is a cumbersome task, as discussed in $\S 1$. We have identified only two objects in the sample that can accurately be clas- sified as FHB stars based on their $\log g$ and $T_{\text {eff }}$ : $23462825-$ 3455590 and $01090411-3307403$. The former (CS 229410050) has been previously confirmed as a halo FHB object by Wilhelm et al. (1999b), who determined the following characteristics for this star: $D=4.2 \mathrm{kpc}, T_{\text {eff }}=8739 \mathrm{~K}, \log g=3.0$, and $[\mathrm{Fe} / \mathrm{H}]=-1.7$. Assuming $M_{J}=+0.80$ (Brown et al. 2004) and using the 2MASS $J$ magnitude, we estimate its distance as $4.6 \mathrm{kpc}$, and the least- $\chi^{2}$ solution indicates $T_{\text {eff }}=8750 \mathrm{~K}$ and $\log g=$ 3.0 (Table 5), in full agreement with Wilhelm et al.'s results. The latter object has not been studied before, but its $J$ magnitude and its classification as a FHB star imply $D=2.1 \mathrm{kpc}$, immersed in the Galactic halo. There also seems to be a third object, 22575321-3250528, that can been classified as FHB based on its low metallicity, deduced from $\log g, T_{\text {eff }}$, and the intensity of its $\mathrm{Ca}$ II $\mathrm{K}$ line.

The remaining objects omitted here and in the two previous sections cannot be reliably classified. Additional observations, especially $U B V$ photometry, may help to figure out $\log g$ and $T_{\text {eff }}$ among the multiple solutions; however, even these new data would leave a significant fraction of the sample unclassified.

\section{CONCLUSIONS}

We carried out a low-resolution spectroscopic survey of hot stars toward the southern Galactic pole, chosen according to their $J H K$ 2MASS color indices. Forty-four stars were observed and had their temperature and surface gravity determined by fitting some of their Balmer features with a grid of model atmospheres. Five objects are classified as subdwarfs, 12 are classified as A stars, and 3 can be reliably classified as FHB objects. The nature of the remaining objects cannot be accurately determined, either because their $\log g$ and $T_{\text {eff }}$ are compatible both with A and FHB types or because of the lack of $U B V$ photometry, which would allow the correct determination of their temperature.

This publication has made use of data products from the Two Micron All Sky Survey, which is a joint project of the University of Massachusetts and the Infrared Processing and Analysis Center, funded by the National Aeronautics and Space Administration and the National Science Foundation. This research has made use of the SIMBAD database, operated at CDS, Strasbourg, France. This work was partially funded by CNPq. R. O., R. D. D. C., and W. J. M. thank FAPESP (grant 2002/08816-5). S. R. is grateful for partial financial support from FAPESP, CNPq, and CAPES.
Altmann, M., Edelmann, H., \& de Boer, K. S. 2004, A\&A, 414, 181

Beers, T. C., Almeida, R., Rossi, S., Wilhelm, R., \& Marsteller, B. 2007, ApJS, 168,277

Beers, T. C., Preston, G. W., \& Shectman, S. A. 1988, ApJS, 67, 461

Beers, T. C., Preston, G. W., Shectman, S. A., Doinidis, S. P., \& Griffin, K. E. 1992, AJ, 103, 267

Beers, T. C., Wilhelm, R., Doinidis, S. P., \& Mattson, C. J. 1996, ApJS, 103, 433 Behr, B. B. 2003, ApJS, 149, 67

Bergeron, P., Fontaine, G., Lacombe, P., Wesemael, F., Crawford, D. L., \& Jakobsen, A. M. 1984, AJ, 89, 374

Bessell, M. S., \& Brett, M. 1988, PASP, 100, 1134

Brown, W. R., Allende Prieto, C., Beers, T. C., Wilhelm, R., Geller, M. J., Kenyon, S. J., \& Kurtz, M. J. 2003, AJ, 126, 1362

Brown, W. R., Geller, M. J., Kenyon, S. J., Beers, T. C., Kurtz, M. J., \& Roll, J. B. 2004, AJ, 127, 1555

Conlon, E. S., Dufton, P. L., Keenan, F. P., \& Leonard, P. J. T. 1990, A\&A, 236, 357

Conlon, E. S., Dufton, P. L., Keenan, F. P., McCausland, R. J. H., \& Holmgren, D. 1992, ApJ, 400, 273

\section{REFERENCES}

Cutri, R. M., et al. 2003, 2MASS All-Sky Catalog of Point Sources (Pasadena: IPAC)

Dorman, B., Rood, R. T., \& O’Connell, R. W. 1993, ApJ, 419, 596

Edelmann, H., Heber, U., Altmann, M., Karl, C., \& Lisker, T. 2005, A\&A, 442, 1023

Flynn, C., Sommer-Larsen, J., \& Christensen, P. R. 1994, MNRAS, 267, 77

Gilmore, G., \& Reid, N. 1983, MNRAS, 202, 1025

Gratton, R. G. 1998, MNRAS, 296, 739

Gray, D. F. 1992, The Observation and Analysis of Stellar Photospheres (2nd ed.; Cambridge: Cambridge Univ. Press)

Green, R. F., Schmidt, M., \& Liebert, J. 1986, ApJS, 61, 305

Harris, H. C., et al. 2003, AJ, 126, 1023

Kilkenny, D. 1995, MNRAS, 277, 920

Kilkenny, D., Heber, U., \& Drilling, J. S. 1988, S. African Astron. Obs. Circ., 12,1

Kilkenny, D., \& Muller, S. 1989, S. African Astron. Obs. Circ., 13, 69

Kilkenny, D., O’Donoghue, D., \& Stobie, R. S. 1991, MNRAS, 248, 664

Kinman, T., \& Castelli, F. 2002, A\&A, 391, 1039

Koornneef, J. 1983, A\&A, 128, 84 
Mitchell, K. J., Saffer, R. A., Howell, S. B., \& Brown, T. M. 1998, MNRAS, 295,225

Morales-Rueda, L., Maxted, P. F. L., Marsh, T. R., Kilkenny, D., \& O’Donoghue, D. 2006, Baltic Astron., 15, 187

Noguchi, T., Maehara, H., \& Kondo, M. 1980, Ann. Tokyo Astron. Obs., 18, 55

Ortiz, R. 2002, Bull. Astron. Soc. Brazil, 21, 41

Pier, J. R. 1982, AJ, 87, 1515 1983, ApJS, 53, 791

Sanduleak, N. 1988, ApJS, 66, 309

Sanduleak, N., \& Pesch, P. 1987, ApJS, 63, 809

Sirko, E., et al. 2004, AJ, 127, 899
Skrutskie, M. F., et al. 1997, in The Impact of Large Scale Near-IR Sky Surveys, ed. F. Garzon et al. (Dordrecht: Kluwer), 25

Sluis, A. P. N., \& Arnold, R. A. 1998, MNRAS, 297, 732

Sommer-Larsen, J., \& Christensen, P. R. 1986, MNRAS, 219, 537

Stetson, P. B. 1991, AJ, 102, 589

Stobie, R. S., Morgan, D. H., Bhatia, R. K., Kilkenny, D., \& O’Donoghue, D. 1987, in IAU Colloq. 95, Second Conference on Faint Blue Stars, ed. A. G. D. Philip, D. S. Hayes, \& J. W. Liebert (Schenectady: Davis), 493

Wilhelm, R., Beers, T. C., \& Gray, R. O. 1999a, AJ, 117, 2308

Wilhelm, R., Beers, T. C., Sommer-Larsen, J., Pier, J. R., Layden, A. C., Flynn, C., Rossi, S., \& Christensen, P. R. 1999b, AJ, 117, 2329

York, D. G., et al. 2000, AJ, 120, 1579 